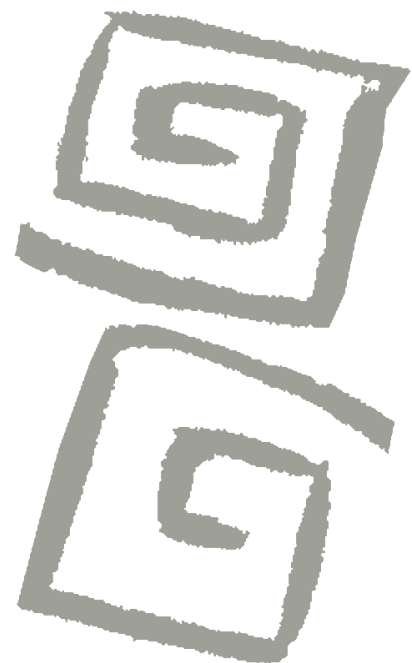

\title{
Anticoncepción y maternidad: Hallazgos de un estudio cuanti-cualitativo con adolescentes de 18 y 19 años de cuatro provincias argentinas
}

\author{
Contraception and maternity: Findings of a quali- \\ quantitative study with 18- and 19-year-olds in four \\ provinces of Argentina
}

Mónica Gogna', Georgina Binstock²

${ }^{1}$ Doctora en Ciencias Sociales. Investigadora Independiente, Consejo Nacional de Investigaciones Científicas y Técnicas (CONICET), Instituto Interdisciplinario de Estudios de Género (IIEGE) Universidad de Buenos Aires (UBA), Argentina. $\triangle$ (iD)

${ }^{2}$ Doctora en Filosofía. Investigadora Independiente, Consejo Nacional de Investigaciones Científicas y Técnicas (CONICET), Centro de Estudios de Población (CENEP), Argentina. $\triangle$ (iD
RESUMEN El artículo analiza los factores que favorecen o dificultan el acceso a los métodos de anticoncepción y a su uso efectivo por parte de adolescentes residentes en las ciudades de Resistencia (Chaco), Posadas (Misiones) y Santiago del Estero (Santiago del Estero) y en las regiones sanitarias V y VII (Buenos Aires). Los datos provienen de una encuesta aplicada a una muestra intencional de 480 adolescentes de 18 y 19 años (sin hijos, con un hijo y con más de un hijo), y de 21 entrevistas semiestructuradas con población de similares características. El 74,2\% de las encuestadas reportó usar un método anticonceptivo "siempre" y un 12,2\% "la mayoría de las veces". Los métodos más utilizados fueron la píldora $(40,7 \%)$ y el preservativo $(37,0 \%)$. Solo el $13,2 \%$ utilizaba un método de mediana o larga duración (inyectable o dispositivo intrauterino). Los principales motivos reportados por quienes "nunca" usaban métodos $(13,6 \%)$ fueron estar buscando un embarazo $(27,6 \%)$ y la oposición de la pareja $(27,6 \%)$. Las entrevistas revelaron dificultades con el uso de los métodos y déficits en la consejería anticonceptiva.

PALABRAS CLAVES Anticoncepción; Adolescentes; Argentina.

\begin{abstract}
The article analyzes the factors that facilitate or hinder access and effective use of contraception by 18- and 19-year-olds living in the cities of Resistencia (province of Chaco), Posadas (province of Misiones) and Santiago del Estero (province of Santiago del Estero) as well as the health regions $\mathrm{V}$ and VII of the province of Buenos Aires. Data comes from a survey applied to a purposeful sample of 480 adolescent females (including respondents without children, with one child, and with more than one child) and 21 semi-structured interviews with a population of the same profile. In response to the survey, $74.2 \%$ reported using contraception "always" and $12.2 \%$ "most of the time." The pill and condoms were the most widely used methods $(41.7 \%$ and $37.0 \%$ respectively). Only $13.2 \%$ used a mid- to long-term method (intrauterine device or injectable contraceptive). The main reasons reported by those who "never" use a method (13.6\%) were: wanting to become pregnant $(27.6 \%)$ and partner refusal $(27.6 \%)$. The interviews revealed difficulties in the use of contraception methods and deficits in contraception counseling.
\end{abstract}

KEY WORDS Contraception; Adolescent; Argentina. 


\section{INTRODUCCIÓN}

En Argentina, como en otros países de la región, el interés por explorar las conductas sexuales y reproductivas de las adolescentes surgió de la preocupación por comprender el fenómeno de la fecundidad adolescente y se profundizó a partir de la epidemia del $\mathrm{VIH} / \mathrm{sida}^{(1)}$.

En la actualidad, este interés sigue vigente, en tanto esta fecundidad es mayoritariamente no deseada, relativamente alta (65 por mil en 2013) en comparación con la de los países vecinos como Uruguay, Chile y Brasil, y ha tenido un incremento del $14,6 \%$ entre 2003 y $2013^{(2)}$. Presenta también importantes diferencias regionales que van desde el 29,8 por mil en la Ciudad Autónoma de Buenos Aires hasta el 91,4 por mil en Misiones, y 90,2 en Formosa ${ }^{(3)}$.

A diferencia de lo ocurrido con la tasa de fecundidad adolescente, se registra un descenso de nacimientos de segundo o mayor orden entre las adolescentes: en los nacimientos de mujeres de entre 18 y 19 años descendió del $31 \%$ al $26 \%$ entre 2001 y 2006 y se mantuvo estable en ese valor hasta 2011. Probablemente, esto se deba a que -como en otros países latinoamericanos- las adolescentes comienzan a usar la anticoncepción de manera más eficaz y sistemática después del nacimiento de su primer hijo(4).

El conocimiento acumulado tras más de dos décadas de investigación académica muestra que la fecundidad y la maternidad en la adolescencia siguen patrones similares a otros países de la región ${ }^{(4,5,6,7)}$. Quienes se convierten en madres en esta etapa de la vida son, en su mayoría, las adolescentes pobres. Aunque persisten diferenciales por clase social, el conocimiento de los métodos anticonceptivos y de las formas de transmisión y prevención del VIH está extendido. No obstante, esto no implica que el uso de métodos sea sostenido o eficaz ${ }^{(8)}$. Visto en perspectiva histórica, el uso de métodos anticonceptivos ha aumentado (especialmente en la iniciación sexual, transición que se ha adelantado ligeramente) pero existen diferenciales según el sexo, la edad y el nivel de instrucción. Las mujeres y adolescentes menores, y quienes viven en condiciones de pobreza están en situación de mayor vulnerabilidad respecto de embarazos no buscados e infecciones sexualmente transmisibles ${ }^{(9,10)}$.

De hecho, la mayor parte de los embarazos de adolescentes no son planificados ${ }^{(2)}$ y esto se mantiene entre generaciones y órdenes de nacimiento(11). Existe evidencia de que las adolescentes mayores, que conviven con su pareja y que están fuera del sistema escolar, son quienes mayoritariamente manifiestan haber buscado el embarazo ${ }^{(12)}$. Después del nacimiento del primer hijo, disminuye el uso del preservativo y aumenta el de anticoncepción hormonal aunque no siempre con resultados satisfactorios ${ }^{(11,12)}$.

Estos datos indican que el acceso que tienen las adolescentes a los servicios de salud reproductiva es un tema en el que aún se puede profundizar mucho ${ }^{(6,8)}$. Sin desconocer que la "cuestión del embarazo planificado o no planificado" es compleja ${ }^{(13)}$ y que las políticas públicas orientadas a prevenir la maternidad en la adolescencia deben ir más allá de la educación sexual y la entrega de métodos anticonceptivos ${ }^{(14)}$.

En Argentina, en el año 2002 se sancionó la Ley 25673 de creación del Programa Nacional de Salud Sexual y Procreación Responsable ${ }^{(15)}$, el cual se reglamenta en marzo de 2003, y en 2006 se sancionó la Ley 26150 de creación del Programa Nacional de Educación Sexual Integral ${ }^{(16)}$, entre otras medidas. En un contexto en el que se han producido importantes cambios legislativos y programáticos tendientes a promover la salud sexual y reproductiva de la población en general y de las y los adolescentes en particular, resulta relevante investigar los factores que favorecen o dificultan el acceso a los métodos de anticoncepción y su uso efectivo desde el punto de vista de las adolescentes. Generar información sobre esta cuestión para poblaciones específicas es fundamental para informar las políticas de salud reproductiva y optimizar la implementación de los programas ${ }^{(6)}$.

Este artículo busca contribuir al conocimiento de esta temática mediante el análisis 
de información sobre la conducta sexual y las intenciones y el comportamiento reproductivo de mujeres de 18 y 19 años que residen en las ciudades capitales de tres provincias del norte argentino y en las regiones sanitarias $\mathrm{V}$ y VII de la provincia de Buenos Aires. Se trata de provincias con las más altas tasas de fecundidad adolescente y una importante proporción de población viviendo en condiciones de pobreza que se atiende en el sector público de salud.

La información proviene de un estudio cuanti-cualitativo más amplio (titulado "Causas y consecuencias del embarazo en la adolescencia en sectores vulnerables") que examinó los factores asociados a la maternidad en la adolescencia prestando especial atención a los determinantes que influyen en que las adolescentes tengan un segundo embarazo/hijo. Producto de esa investigación se ha publicado un artículo sobre los entornos en que ocurren los embarazos en la adolescencia ${ }^{(11)}$ y otro sobre la iniciación sexual ${ }^{(17)}$. El presente artículo reporta datos inéditos de un módulo especial aplicado a adolescentes de 18 y 19 años.

\section{MÉTODO}

La metodología del estudio marco consistió en la aplicación de una encuesta a una muestra no representativa de 1.571 adolescentes y jóvenes de 18 a 24 años, en las ciudades de Resistencia (Chaco), Posadas (Misiones), Santiago del Estero (Santiago del Estero) y en las regiones sanitarias $\mathrm{V}$ y VII (Buenos Aires). Además, se realizaron 45 entrevistas semiestructuradas a mujeres de este grupo de edad en el año 2012. Las provincias seleccionadas se encuentran entre aquellas con las tasas de fecundidad adolescente más altas y la mayor proporción de jóvenes entre 15 y 19 años que han tenido más de un hijo. La provincia de Buenos Aires, por su parte, es la jurisdicción con el mayor número de madres adolescentes, tanto primíparas como multíparas. Las regiones sanitarias de esta provincia fueron seleccionadas en conjunto con las autoridades del área en función de prioridades programáticas.

Las adolescentes y jóvenes fueron contactadas en centros de salud y hospitales (previa autorización de las respectivas autoridades) cuando concurrían a realizar consultas propias (por ejemplo, consulta ginecológica, control de embarazo, entre otras) o llevaban a sus hijos a controles médicos (salas de salud, hospitales pediátricos). Se las invitaba a participar de la encuesta o de la entrevista, manifestándole que su participación era voluntaria, que podía interrumpir la encuesta o la entrevista si así lo deseaba o no contestar alguna pregunta y que todo ello sería confidencial; $y$, si aceptaba, se le solicitaba su consentimiento informado. El protocolo de investigación fue sometido a la aprobación de un Comité de Ética ad hoc integrado por tres investigadores (del Instituto de Investigaciones Gino Germani, Facultad de Ciencias Sociales, Universidad de Buenos Aires, y del Centro de Estudios de Población). Su dictamen favorable fue elevado oportunamente a la Organización Mundial de la Salud, cuya contribución fue decisiva para el desarrollo del estudio.

Dados los objetivos del estudio y a los fines de garantizar el número de casos para los análisis sustantivos, se establecieron cuotas: mujeres que no tuvieron ningún hijo durante la adolescencia, mujeres que tuvieron un hijo y mujeres que tuvieron dos o más hijos durante la adolescencia.

La encuesta relevó el contexto de crianza y la trayectoria educativa y laboral; las edades de la menarca y el inicio de relaciones románticas; la edad, contexto y circunstancias de la iniciación sexual y el conocimiento y uso de métodos anticonceptivos; y reconstruyó la historia reproductiva de las jóvenes. Las entrevistas indagaron, entre otros aspectos, las motivaciones para el uso de métodos, las experiencias con la anticoncepción y los significados otorgados a la maternidad.

Además, para las adolescentes de 18 y 19 años, la encuesta incluyó un módulo especial con preguntas acerca de la actividad sexual reciente y el cuidado anticonceptivo al momento de la encuesta, el conocimiento y uso de la anticoncepción hormonal de 
emergencia y el nivel de preocupación que las jóvenes tenían de quedar embarazadas y de contraer $\mathrm{VIH} /$ sida.

Este artículo centra la atención en estas adolescentes, sobre la base de información inédita de dicho módulo, y de 21 entrevistas realizadas a adolescentes "tardías", es decir, de 18 y 19 años, que exploraron el conocimiento, la aceptabilidad y las razones de uso/ no uso de métodos anticonceptivos así como los diferentes posicionamientos respecto de la maternidad. Las entrevistas tuvieron una duración de aproximadamente 40 minutos, fueron grabadas previo consentimiento informado de las adolescentes y transcriptas (se utilizan nombres de fantasía para resguardar el anonimato). El material fue codificado sobre la base de descriptores derivados del marco conceptual y de códigos "emergentes" y, a partir de allí, se realizó un análisis destinado a identificar patrones y relaciones entre categorías significativas ${ }^{(18)}$. El interés por el comportamiento de las Ilamadas adolescentes "tardías" obedece a que este grupo concentra entre la mitad y el $65 \%$ de los nacimientos de madres adolescentes ${ }^{(19)}$.

\section{RESULTADOS}

Respecto del perfil sociodemográfico de la muestra (Tabla 1), el 21,5\% tenía primaria completa o menos, el 51,9\% secundaria incompleta (de las cuales el 16,3\% asistía al momento de la encuesta) y el $26,7 \%$ secundaria completa o más. Estos datos muestran una situación educativa claramente más desventajosa que la registrada por la Encuesta Nacional de Salud Sexual y Reproductiva (ENSSyR) de 2013 (según la cual el 44\% de las adolescentes de 18 y 19 años tenía secundaria completa o más).

El 92,1\% de las encuestadas se había iniciado sexualmente, la mayoría $(79,8 \%)$ con el novio, siendo minoritaria la proporción que lo hizo con un amigo $(11,1 \%)$ o conocido $(6,6 \%)$. El $75,3 \%$ de las sexualmente iniciadas reportó haber utilizado preservativo en su primera relación. Entre aquellas que
Tabla 1. Características sociodemográficas de las adolescentes de 18 y 19 años encuestadas $(n=480)$. Ciudades de Resistencia (Chaco), Posadas (Misiones), Santiago del Estero (Santiago del Estero) y regiones sanitarias V y VII (Buenos Aires), 2012.

\begin{tabular}{|c|c|c|}
\hline Características sociodemográficas & $\mathrm{n}$ & $\%$ \\
\hline \multicolumn{3}{|l|}{ Nivel de instrucción y asistencia } \\
\hline Primaria incompleta o menos & 35 & 7,2 \\
\hline Primaria completa & 68 & 14,2 \\
\hline Secundaria incompleta & 249 & 51,9 \\
\hline No asiste & 171 & 35,6 \\
\hline Asiste & 78 & 16,3 \\
\hline Secundaria completa y más & 128 & 26,7 \\
\hline No asiste & 107 & 22,3 \\
\hline Asiste & 21 & 4,4 \\
\hline \multicolumn{3}{|l|}{ Asistencia escolar } \\
\hline Asiste & 103 & 21,5 \\
\hline No asiste & 377 & 78,5 \\
\hline \multicolumn{3}{|l|}{ Situación conyugal } \\
\hline Soltera & 252 & 52,5 \\
\hline Casada & 8 & 1,7 \\
\hline Unida & 195 & 40,6 \\
\hline Separada & 25 & 5,2 \\
\hline \multicolumn{3}{|l|}{ Trabajo } \\
\hline Trabaja & 80 & 16,7 \\
\hline No trabaja actualmente & 195 & 40,6 \\
\hline Nunca trabajó & 205 & 42,7 \\
\hline \multicolumn{3}{|l|}{ Sexualmente iniciada } \\
\hline Sí & 442 & 92,1 \\
\hline No & 38 & 7,9 \\
\hline \multicolumn{3}{|c|}{ Embarazada al ser encuestada $(n=442) *$} \\
\hline Sí & 126 & 28,6 \\
\hline No & 316 & 71,4 \\
\hline \multicolumn{3}{|l|}{ Hijos nacidos vivos $(\mathrm{n}=442)^{*}$} \\
\hline Ninguno & 242 & 54,8 \\
\hline No embarazada & 147 & 33,2 \\
\hline Embarazada & 95 & 21,6 \\
\hline 1 hijo & 168 & 38 \\
\hline No embarazada & 29 & 6,6 \\
\hline Embarazada & 139 & 31,4 \\
\hline 2 hijos o más & 32 & 7,3 \\
\hline No embarazada & 30 & 6,8 \\
\hline Embarazada & 2 & 0,5 \\
\hline
\end{tabular}

Fuente: Elaboración propia. *Entre sexualmente iniciadas.

aún no habían debutado sexualmente $(7,9 \%)$ el principal motivo había sido el "no haber encontrado a la persona adecuada" $(43,9 \%)$ (17). En cuanto a la situación conyugal, el $52,5 \%$ se declaró soltera, el $40,6 \%$ unida, el $1,7 \%$ casada y el $5,2 \%$ separada. 
El 54,8\% no tenía hijos (el 21,6\% estaba embarazada al momento de la encuesta y el $33,2 \%$ restante no), el 38,0\% tenía un hijo y el $7,3 \%$ dos o más. Además, el $16,7 \%$ de las adolescentes trabajaba al momento de la encuesta y el $42,7 \%$ nunca había trabajado.

Un $11,4 \%$ de las adolescentes sexualmente iniciadas no había tenido relaciones sexuales en los tres meses previos a la encuesta. Entre quienes tuvieron actividad sexual, prácticamente tres de cada cuatro reportó una única pareja sexual. El $77,4 \%$ de las adolescentes encuestadas nunca había tenido relaciones sexuales en situación de consumo excesivo de alcohol y, para el $14,1 \%$, esto había sucedido "muy pocas veces".

El 70,1\% de las adolescentes había consultado y/o recibido información sobre métodos anticonceptivos por el sistema público de salud (hospitales, centros de salud), y la mayoría de ellas $(67,5 \%)$ había recibido algún método, mayoritariamente píldoras anticonceptivas. De hecho, las entrevistas semiestructuradas revelaron cierto consenso entre las jóvenes en el sentido de que actualmente la información es más accesible que antes.

\begin{abstract}
Ahora se habla en la escuela, se habla en los hospitales, en todos lados. Es una cosa normal cuidarse (Daiana, 18 años, dos hijos, AMBA)

Ya sabía. Yo digo que todos los jóvenes sabemos eso pero siempre ocurren accidentes. No puede una chica venir y decir que no sabe. Yo digo que todos saben. (Marianela, 18 años, un hijo, AMBA)
\end{abstract}

Con respecto a la frecuencia de uso de métodos entre quienes tuvieron relaciones sexuales en los últimos tres meses (y no estaban embarazadas), el 74,2\% reportó usar "siempre" y, un 12,2\%, "la mayoría de las veces", lo que resulta consistente con resultados de estudios previos en nuestro país ${ }^{(9)}$ y con información provista, entre otros, por García y Koyama para Brasil(20). Entre las encuestadas que no se cuidaban de manera consistente $(13,6 \%)$, los principales motivos fueron estar buscando un embarazo $(27,6 \%)$ y la oposición de la pareja $(27,6 \%)$. El resto de los motivos refieren a la poca actividad sexual, el temor a los efectos secundarios de los métodos, la falta de dinero para comprarlos, la lejanía del lugar donde obtener el método. Que la gestión de la contracepción dependa de la capacidad de negociación con la pareja sexual habla de una permanencia de la jerarquía de género -también observada por Reis Brandão y Heilborn ${ }^{(21)}$ - que requiere ser abordada tanto en la educación sexual como en la consejería anticonceptiva.

Las entrevistas semiestructuradas, a su vez, arrojaron luz sobre las motivaciones detrás de la búsqueda de un embarazo. Ellas son consistentes con lo reportado por la bibliografía: la maternidad puede ser parte de un proyecto de vida, una especie de "pasaporte" para entrar en la vida adulta y una fuente de confianza en sí mismas ${ }^{(22,23)}$. Las adolescentes son conscientes de que esta no es la interpretación más habitual, tal como se desprende de la justificación que diera Carolina (18 años, un hijo, AMBA) al explicitar que su embarazo había sido buscado: "No tiene nada de malo". Oriana (19 años, embarazada, Posadas) manifestó que quería tener un hijo porque le encantan los chicos y que le "parecía que ya era hora de formar una familia". Romina (19 años, un hijo, Resistencia), por su parte, argumentó que no tenía hermanos y que "sentía que quería ser madre". El testimonio de Jessica (19 años, un hijo, embarazada, Posadas), quien enfrentó las críticas de su madre y de su suegra, muestra que muchas veces los varones son copartícipes de la decisión: "nosotros queríamos..., queríamos tener esa experiencia de cómo es tener un hijo".

Los métodos más utilizados fueron la píldora $(40,7 \%)$ y el preservativo $(37,0 \%)$. En menor medida, el inyectable $(8,2 \%)$, la "doble protección" de píldora y preservativo $(8,2 \%)$ y el dispositivo intrauterino $(5,4 \%)$. Estos datos son consistentes con los que arroja la ENSSyR que muestra que, entre las adolescentes, a mayor edad es más frecuente el uso de métodos de control femenino ${ }^{(24)}$. No obstante, las entrevistadas reportaron también la adopción de métodos poco seguros como 
el coito interrumpido, y el uso discontinuo del preservativo y "olvidos" en la toma de la píldora anticonceptiva.

El típico ese de que terminás afuera... Llegó igual (Daiana, 18 años, un hijo y embarazada, AMBA)

[¿Te hablaron alguna vez de métodos?] Sí, ya lo sabía. Lo que pasa es que en la adolescencia... "aj!, no me va a pasar" (Yohana, 19 años, dos embarazos durante la adolescencia, Resistencia).

Asimismo, los relatos también pusieron de manifiesto dificultades en el diálogo entre las adolescentes y los profesionales de la salud respecto de los métodos, sus efectos y condiciones de uso. Tanto a Magalí (18 años, un hijo, AMBA) como a Carolina (18 años, un hijo, AMBA) las ginecólogas les recomendaron la píldora, aunque en la entrevista ambas manifestaron reparos respecto de este método. Magalí estaba pensando en cambiar por el inyectable ("me olvido siempre" [de tomar el anticonceptivo oral]) y Carolina se estaba cuidando con preservativo por el mismo motivo. Solo una entrevistada había logrado reemplazar la píldora por otro método de alta eficacia y mayor comodidad (el inyectable). Varias entrevistadas reportaron que quedaron embarazadas cuando dejaron la píldora, método que les "hacía mal" y al que le atribuyeron provocar dolor de estómago, alergias, sarpullido y aumento de peso.

Dado que mayoritariamente se trataba de adolescentes que habían tenido contacto con los servicios de salud, sus embarazos no buscados ponen en cuestión la oportunidad y eficacia de la consejería anticonceptiva recibida. ¿Recurrieron las entrevistadas a la anticoncepción hormonal de emergencia (AHE), "última barrera" cuando el método anticonceptivo de uso regular falló o cuando no se usó un anticonceptivo? La pregunta resultaba relevante pues estudios previos indicaban que este método se difunde básicamente a través de fuentes no médicas ${ }^{(25)}$ y que los profesionales son reticentes a informar $y$ prescribirla ${ }^{(26)}$.
El conocimiento de la AHE no estaba tan generalizado como el del preservativo y la píldora: algo más del 70\% de las encuestadas la conocía y, de ellas, una de cada cuatro la había utilizado el año anterior. El conocimiento es algo inferior pero el uso bastante similar al reportado en un estudio realizado en el Uruguay ${ }^{(27)}$. Las entrevistas semiestructuradas indicaron que quienes las habían usado no habían tenido inconvenientes. Caty lo resumió en una corta frase "Vas a la farmacia y la comprás nomás". Jaqui, por su parte, se había enterado de la existencia de la AHE en la escuela, a través de una profesora que les explicó cómo funcionaba y que se podía tomar "en situaciones riesgosas". No obstante, antes de tomarla "leyó todo" por Internet. Otras entrevistadas también habían conocido este método a través de amigas, compañeras de colegio y novios. En consonancia con estudios previos, el sector salud no aparece en sus relatos como fuente de información acerca de este método.

Finalmente, el $77,1 \%$ de las encuestadas reportó haber recibido información acerca del $\mathrm{VIH} /$ sida pero solo una minoría $(28,4 \%)$ conocía la "doble protección". Al indagar si el VIH/sida constituía para ellas un motivo de preocupación, la mitad de las encuestadas indicó que le preocupaba "mucho" (resaltaron la "gravedad" de la infección), a un cuarto le preocupaba "poco" y a otro cuarto "nada" (en general se consideró a la pareja "estable" como una forma de protección). Estas proporciones no difieren sustantivamente de las que se obtienen cuando se indaga acerca de la preocupación por un embarazo: al 52,6\% le preocupaba "mucho" quedar embarazada, al $17,2 \%$ "poco" y para el $30,2 \%$ esa posibilidad no era motivo alguno de preocupación. Si se tiene en cuenta la condición de maternidad, las adolescentes madres reportan con mayor frecuencia que les preocupa "mucho" quedar embarazadas en comparación con las adolescentes que no son madres $(64,5 \%$ versus $46,1 \%$ ). Esta pregunta no se le formuló a las embarazadas y a las puérperas (entrevistadas en el posparto).

Como era de esperarse, quienes manifestaron preocupación ante un eventual 
embarazo reportaron cuidarse en mayor proporción. Entre quienes en los últimos tres meses no habían usado anticoncepción "siempre", a dos tercios les preocupaba "poco o nada" un embarazo y al tercio restante le preocupaba "mucho".

Las respuestas a una pregunta abierta ayudaron a comprender a quiénes y por qué les resultaba (o no) preocupante un embarazo. Un grupo de entrevistadas se manifestó confiada en la eficacia del cuidado anticonceptivo. Sus testimonios trasuntan seguridad: "hay formas de cuidarse", "nos cuidamos los dos"; "estoy segura de que me cuido bien"; "me siento bien con este método"; "tengo bastante información sobre cómo cuidarme". Otro grupo especificó que un embarazo no resultaría problemático porque tenía una pareja estable, trabajo y dinero para mantener un hijo o porque ya tenía un hijo y otro no representaría una gran diferencia.

Las adolescentes que manifestaron temor frente a un eventual embarazo explicitaron que sería un obstáculo para sus planes ya que estaban estudiando o querían continuar los estudios y disfrutar de salidas. Un segundo conjunto de respuestas aludía a la edad: "soy chica" constituye un patrón que condensa el sentimiento de no tener aún la edad adecuada para ser madre. La frase usualmente fue acompañada de una pequeña explicación: no estar preparada, no "verse" con un hijo, no querer ser una carga para la madre o considerarlo mucha responsabilidad en este momento de su vida. Expresiones similares fueron vertidas en las entrevistas semiestructuradas:

Te alegra la vida capaz, pero soy muy chica. No pueden salir. $Y$ a veces no tienen para la leche. Le tienen que prestar. (Daniela, 19 años, sin hijos, Resistencia)

El hijo es una bendición pero algunas están en mala situación. Es mejor si un hijo viene con trabajo, casa. (Estefi, 19 años, sin hijos, Posadas)
[¿Qué te motiva a cuidarte?] Es poder tener un lugar digno para el día que tenga un hijo... Una casa grande, un buen trabajo. A lo de mi mamá no quisiera yo ir a vivir con una criatura. A lo de él tampoco. Tener mi lugar propio.

(Nancy, 19 años, sin hijos, AMBA)

Entre las adolescentes que ya tenían un hijo, el temor a un nuevo embarazo se debía a que, por su corta edad, el hijo/a requería atención y cuidados o a la falta de trabajo o dinero para mantener otro. Otros motivos de preocupaciones fueron haber sufrido durante el embarazo previo, tener un hijo enfermo y no tener pareja.

\section{DISCUSIÓN}

Los resultados de nuestro estudio corresponden a adolescentes mayores, de 18 y 19 años, que viven en situaciones de vulnerabilidad según surge de la comparación con los datos de la ENSSyR 2013 (tienen un nivel educativo más bajo, menor conocimiento de anticonceptivos, y menor edad y uso de métodos en la iniciación sexual) ${ }^{(24)}$.

Nuestros hallazgos confirman muchas de las tendencias ya identificadas por estudios previos en Argentina y los de otros países del Cono Sur ${ }^{(7,10,28)}$. El conocimiento de métodos anticonceptivos está bastante generalizado y el uso de métodos, relativamente alto en la iniciación sexual, decae posteriormente. La píldora y el preservativo son los métodos más usados, y se registra una pequeña proporción de adolescentes que utiliza métodos de mediana o larga duración. La anticoncepción hormonal de emergencia tiene un nivel de conocimiento menor que los otros métodos, y la información acerca de la misma proviene fundamentalmente de fuentes no médicas.

A su vez, el estudio arrojó luz acerca de algunos factores que facilitan o dificultan el uso de métodos anticonceptivos entre las adolescentes mayores. Entre los facilitadores, sin duda, se destaca la motivación. El uso es más sistemático entre aquellas adolescentes 
para quienes el embarazo constituye una "preocupación" (sea porque interfiere con sus estudios, no se consideran preparadas, quisieran postergar la maternidad hasta contar con más recursos económicos o porque ya tienen hijos). Entre los obstáculos, se destacaron la oposición de las parejas masculinas, la escasa frecuencia de las relaciones sexuales y el temor a los efectos adversos de los anticonceptivos. Aunque de diverso orden, los tres factores ponen en evidencia la necesidad de que la consejería anticonceptiva atienda a la multiplicidad de factores que inciden en la elección y continuidad de un método anticonceptivo (el contexto de la relación, la vivencia de la sexualidad, los efectos secundarios, etc.). La desigualdad entre los géneros respecto de las decisiones reproductivas hace necesario que los programas de salud reproductiva capaciten al personal en la perspectiva de género y derechos de modo que comprendan la importancia de incorporar a los varones y promover la autonomía de las usuarias para alcanzar una prevención más eficaz.

La consejería anticonceptiva, clave para la accesibilidad al método, parece constituir un reto para los profesionales dada la variabilidad de experiencias registradas. Mientras algunas adolescentes manifestaron sentimientos de confianza y autoeficacia respecto de sus prácticas anticonceptivas, otras no se sintieron habilitadas a expresar sus dudas, temores y/o preferencias. Incluso, en algunos casos, las entrevistadas reportaron que el método que se les ofreció no estaba de acuerdo con sus necesidades y deseos.

Finalmente se identificaron problemas con el uso de los métodos más frecuentemente utilizados por las adolescentes. En el caso de la píldora, las dificultades se relacionan con su modalidad (ingesta diaria) y con los efectos experimentados o atribuidos. En el caso del preservativo, se reportó uso esporádico o incorrecto.

\section{RECOMENDACIONES}

Tal como lo indica un estudio sobre prevención del embarazo adolescente en países de ingresos medios y bajos ${ }^{(29)}$ para que las adolescentes que no quieren ser madres aún logren una conducta anticonceptiva sostenida y eficaz es necesario que las y los profesionales aseguren el acceso a un método apropiado y aceptable y al conocimiento necesario para usarlo adecuadamente.

Dadas las dificultades que presenta la anticoncepción hormonal, es deseable diversificar la oferta de métodos incluyendo otras opciones de mediano y largo plazo tales como el dispositivo intrauterino, los implantes y los inyectables. Ello supone su entrega sin barreras (tiempos de espera, estudios innecesarios), el conocimiento -transmitido en lenguaje accesible- que permita tomar decisiones informadas y que las usuarias puedan acceder con facilidad a la remoción si así lo desearan ${ }^{(30)}$. Asimismo, dadas las dificultades con el uso de la píldora y el preservativo y el desafío que supone una conducta anticonceptiva sostenida en el marco de relaciones de género desiguales, resulta clave que las adolescentes puedan acceder a la anticoncepción hormonal de emergencia en tiempo y forma. Esta es, aún, una deuda pendiente de los servicios de salud para con las y los adolescentes.

\section{AGRADECIMIENTOS}

El artículo reporta resultados de un estudio más amplio que fue financiado por la Organización Mundial de la Salud (CP2 09 Argentina: "Causes and consequences of pregnancy during adolescence among impoverished sectors in Argentina"). El módulo especial para adolescentes de 18 y 19 años -que dio origen a este artículofue realizado con apoyo del Fondo de Población de las Naciones Unidas (UNFPA) de Argentina. Agradecemos en las personas de lqbal Shah, Venkatraman Chandra-Mouli y Eleonor Faur a las agencias donantes. También a las coordinadoras provinciales del Programa Nacional de Salud Integral en la Adolescencia, que facilitaron el acceso 
al campo: Liliana Ensisa (Chaco), Cecilia Chami y Gisella Chamud (Santiago del Estero) y Alicia Díaz (Misiones). A Lidia Schiavoni (antropóloga, Universidad Nacional de Misiones), Claudia Stilman (asistente de investigación), Inés Ibarlucía y Mariana Kielmanovich (entrevistadoras). Y muy especialmente a las adolescentes y jóvenes que participaron del estudio.

\section{REFERENCIAS BIBLIOGRÁFICAS}

1. Pantelides EA. La fecundidad adolescente y sus consecuencias: Clase social, género y conducta sexual adolescente. En: Salud Reproductiva: Nuevos Desafíos. Lima: Universidad Peruana Cayetano Heredia, IEPO-PROSAR; 1996. p. 239-251.

2. Programa Nacional de Salud Integral en la Adolescencia, Ministerio de Salud, UNICEF. Situación de salud de los y las adolescentes en la Argentina. Buenos Aires: MSAL, UNICEF; 2016.

3. Ministerio de Salud de la Nación, Dirección de Estadísticas e Información de Salud. Tasa de fecundidad adolescente, tasa temprana y tasa tardía por cada 1.000 mujeres, según jurisdicción de residencia. Indicadores seleccionados de salud para la población de 10 a 19 años: Año 2013. 2015;149:13.

4. Rodríguez Vignoli J. Fecundidad adolescente en América Latina: una actualización. En: Cavenaghi S, Cabella W, (ed). Comportamiento reproductivo y fecundidad en América Latina: una agenda inconclusa (Serie e-Investigaciones No3). Río de Janeiro: FNUAP, ALAP; 2014. p. 33-65.

5. Guttmacher Institute, IPPF. In brief: Facts on the sexual and reproductive health of adolescent women in the developing world. London: Guttmacher Institute, IPPF; 2010.

6. Vogel JP, Pileggi-Castro C, Chandra-Mouli V, et al. Millennium Development Goal 5 and adolescent: looking back, moving forward. Archives of Disease in Childhood. 2015;100(Suppl 1):S43-S47.

7. Plan Internacional, UNICEF. Vivencias y relatos sobe el embarazo en adolescentes: Una aproximación a los factores culturales, sociales y emocionales a partir de un estudio en seis países de la región. Panamá: Plan Internacional, UNICEF; 2014.

8. Pantelides EA, Manzelli H. Investigación reciente sobre sexualidad y salud reproductiva de las/los adolescentes en América latina: qué hemos alcanzado, qué falta hacer, cuáles son nuestras falencias. En: Cáceres C, Cueto M, Ramos M, VaIlenas S, (ed). La salud como derecho ciudadano en América Latina. Lima: UPCH Fondo Editorial; 2003. p. 73-87.

9. Kornblit A, Mendes Diz A, Adasko D. Prácticas sexuales de jóvenes escolarizados en la Argentina: relevancia de su conocimiento para la educación sexual. En: López E, Pantelides EA, (ed.). Aportes a la investigación social en salud sexual y reproductiva. Buenos Aires: CENEP, CEDES, AEPA, UNFPA; 2005. p. 83-104.

10. Fundación Huésped, UNICEF. Conocimientos, actitudes y prácticas en VIH y salud sexual y reproductiva (SSR) y uso de tecnologías de la información (TIC) entre adolescentes de Argentina. Buenos Aires: UNICEF, Fundación Huésped; 2012.

11. Binstock G, Gogna M. Entornos del primer y segundo embarazo en la adolescencia en Argentina. En: Cavenaghi S, Cabella W, (ed.). Comportamiento reproductivo y fecundidad en América Latina: una agenda inconclusa (Serie eInvestigaciones $\mathrm{N}^{\circ}$ 3). Río de Janeiro: FNUAP, ALAP; 2014. p. 167-185.

12. Gogna M, Fernández S, Zamberlin N. Historias reproductivas, escolaridad y contexto del embarazo: hallazgos de la encuesta a puérperas. En: Gogna M, (ed.). Embarazo y maternidad en la adolescencia: Estereotipos, evidencias y propuestas para políticas públicas. Buenos Aires: CEDES, UNICEF, Ministerio de Salud de la Nación; 2005. p. 251-284.

13. Greco A. Las voces acalladas en la maternidad: Los controles prenatales ausentes o inadecuados en la perspectiva de las mujeres de sectores populares. Buenos Aires: CEDES, FLACSO; 2008.

14. Fletcher A, Harden A, Brunton G, Oakley A, Bonell C. Interventions addressing the social determinants of teenage pregnancy. Health Education. 2007;108(1):29-39.

15. Argentina. Ley 25673 Programa Nacional de Salud Sexual y Procreación Responsable [Internet]. 2002 [citado 12 ago 2015]. Disponible en: http:// tinyurl.com/ztcpokf.

16. Argentina. Ley 26150 Programa Nacional de Educación Sexual Integral [Internet]. 2006 [citado 12 ago 2015]. Disponible en: http://tinyurl.com/ jccdr2q.

17. Binstock G, Gogna M. La iniciación sexual entre mujeres de sectores vulnerable en cuatro provincias argentinas. Sexualidad, Salud y Sociedad. Revista Latinoamericana. 2015;(20):113-140. 
18. Patton MQ. Qualitative research \& evaluation methods. 3a ed. Thousand Oaks: Sage; 2002.

19. Binstock G, Pantelides EA. La fecundidad adolescente hoy: diagnóstico sociodemográfico. En: Gogna M, (ed.). Embarazo y maternidad en la adolescencia. Estereotipos, evidencias y propuestas para políticas públicas. Buenos Aires: CEDES, UNICEF, Ministerio de Salud de la Nación; 2005. p. 77-112.

20. García S, Koyama M. Longevidade sexual e práticas sexuais despro $\neg$ tegidas: desafios para a promoção da saúde sexual e reprodutiva das mulheres. En: Brasil, Ministério da Saúde. Pesquisa Nacional de Demografia e Saúde da Criança e da Mulher, PNDS 2006: dimensões do processo reprodutivo e da saúde da criança. Brasília: Ministério da Saúde; 2009.

21. Reis-Brandão E, Heilborn ML. Sexualidade e gravidez em jovens de camadas medias. Cadernos de Saúde Pública. 2006;22(7):1421-1430.

22. Nauar-Pantoja AL. "Ser alguém na vida": uma análise sócio-antropológica da gravidez/maternidade na adolescência, em Belém do Pará, Brasil. Cadernos de Saúde Pública. 2003;19(Suppl 2):S335-S343.

23. Mazzini MLH, Alves ZMMB, Silva MRS, Sagim MB. Mães adolescentes: a construção de sua identidade materna. Ciência, Cuidado e Saúde. 2008;7(4):493-502.

24. Binstock G, Pantelides EA. Las conductas sexuales y reproductivas de los adolescentes: La iniciación sexual, Resultados de la ENSSyR 2013. Ponencia presentada a las XIII Jornadas Argentinas de Estudios de Población. Salta, 16-18 de septiembre de 2015 .

25. Pecheny $M$, Tamburrino MC. ¿"La palabra lo dice"?: Interpretaciones cruzadas y obstáculos al acceso a la anticoncepción de emergencia. Sexualidad, Salud y Sociedad - Revista Latinoamericana. 2009;(1):158-176.

26. Gogna M. Salud reproductiva y adolescencia: Una mirada de género sobre los conflictos y dilemas de las y los profesionales en un contexto en transición [Tesis de doctorado]. Buenos Aires: Universidad de Buenos Aires; 2008.

27. MYSU, Observatorio Nacional de Género y Salud Sexual y Repro $\neg$ ductiva. Estudio 2011-2012: Necesidades y demandas en salud sexual y reproductiva en mujeres adolescentes uruguayas. Montevideo: MYSU; 2013.

28. Fondo de Población de las Naciones Unidas. Fecundidad y maternidad adolescente en el Cono Sur: Apuntes para la construcción de una agenda común [Internet]. 2016 [citado 12 ago 2015]. Disponible en: http://tinyurl.com/zmull34.

29. Chandra-Mouli V, McCarraher DR, Phillips SJ, Williamson NE, Hainsworth G. Contraception for adolescents in low and middle income countries: needs, barriers, and access. Reproductive Health. 2014;11(1):1. doi: 10.1186/1742-4755-11-1.

30. Manchikanti-Gomez A, Fuentes L, Allina A. Women or LARC first?: Reproductive autonomy and the promotion of long-acting reversible contraceptive methods. Perspectives on Sexual and Reproductive Health. 2014;46(3):171-175.

\section{FORMA DE CITAR}

Gogna M, Binstock G. Anticoncepción y maternidad: Hallazgos de un estudio cuanti-cualitativo con adolescentes de 18 y 19 años de cuatro provincias argentinas. Salud Colectiva. 2017;13(1):63-72. doi: 10.18294/sc.2017.990.

Recibido: 20 de mayo de 2016 | Versión final: 20 de octubre de 2016 | Aprobado: 6 de febrero de 2017

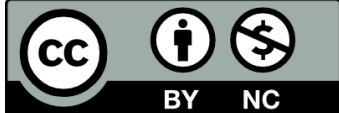

Este obra está bajo una licencia de Creative Commons Reconocimiento-NoComercial 4.0 Internacional. Reconocimiento - Permite copiar, distribuir y comunicar públicamente la obra. A cambio, se debe reconocer y citar al autor original. No Comercial - Esta obra no puede ser utilizada con finalidades comerciales, a menos que se obtenga el permiso. 